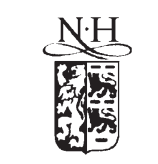

ELSEVIER
Journal of International Money and Finance 20 (2001) 419-438
Journal of

International

Money

and Finance

\title{
The lifetime of a unilateral target zone: some extended results
}

\author{
Simon Broome * \\ Department of Economics, NUI, Maynooth, Co. Kildare, Ireland
}

\begin{abstract}
In Dumas and Svensson (Journal of International Economics 36 (1994) 467) the lifetime of a Krugman (Quarterly Journal of Economics 106 (1991) 669-682) type target zone model is found to be extremely long. This paper shows that the lifetime of the identical target zone regime can be much lower when real disturbances occur. In particular, the mean lifetime is found to be very low when real disturbances occur and the real exchange rate elasticity of the demand for domestic goods is very low. It is also shown that allowing for monetary disturbances to have real effects has very little effect on the lifetime. The results indicate the conditions under which real disturbances might trigger currency crises. (C) 2001 Elsevier Science Ltd. All rights reserved.
\end{abstract}

JEL classification: E42; F31; F41

Keywords: Currency crises; Expected lifetime; Real and monetary shocks

\section{Introduction}

Managed exchange rate regimes last only for finite periods of time before they are either realigned or abandoned. Dumas and Svensson (1994) examine the role of central bank reserves in determining this 'lifetime' when a single central bank attempts to operate an exchange rate target zone. Central bank reserves play a role in exchange rate crises only when political considerations prevent the implementation of unpopular policies. For example, in the first generation speculative attack model of Krugman (1979) and Flood and Garber (1984) a crisis occurs because the central

* Tel.: +353-1-7084567.

E-mail address: simon.j.broome@may.ie (S. Broome). 
bank finances a fiscal deficit by expanding domestic credit. In the absence of any offsetting changes the money supply expands and the exchange rate depreciates. To stabilize the money supply and fix the exchange rate the central bank buys up the excess domestic currency using foreign reserves. Eventually the stock of foreign reserves falls to a critically low threshold at which a speculative attack is triggered wiping out the remaining reserves. If the government were able to eliminate the deficit the collapse could be avoided indefinitely. In the second generation models pioneered by Obstfeld (1986), ${ }^{1}$ an optimizing policy maker abandons the fixed rate in response to mounting unemployment. If the government were unconcerned by rising unemployment the domestic interest rate could be raised sufficiently to compensate for any expected depreciation thereby averting the crisis. In practice, domestic interest rate rises are constrained by the government's desire to limit the rise in unemployment. In these second generation models the stock of reserves does not appear at all, but the ceiling on the interest rate defines an equivalent floor on the money supply and reserves. Thus if implicit constraints prevent the exclusive focus of monetary policy on the target zone limited reserves will play a role in determining its expected lifetime. Assuming that there are such implicit constraints, Dumas and Svensson examine the role of reserves in the lifetime of an exchange rate target zone. The central result of their work is that the lifetime is extremely long. In the example of a $10 \%$ yearly drift of the fundamental and an initial stock of reserves $100 \%$ above the critical minimum, the expected lifetime of a target zone of $\pm 1.5 \%$ is over 10.7 years. In addition, the model predicts the bandwidth itself has only a very minor effect on the lifetime. Simple division shows that the lifetime of a fixed rate in the above case is equal to 10 years. With no drift but a standard deviation of $10 \%$ per year the expected lifetime is a remarkable 120 years. The major determinants of the lifetime are the stock of reserves and the size of the drift in the fundamental. These results suggest that a narrow target zone ought to be an extremely robust structure.

This paper extends the work of Dumas and Svensson by attempting to illustrate the effects of varying the structure of the economy. The basic idea is that whilst a monetary disturbance can always be offset by a one for one change in the money supply, re-establishing equilibrium after a real disturbance may prove to be much more difficult, particularly if domestic production concentrates on differentiated products with a low real exchange rate elasticity of demand. In such a scenario movements in real fundamentals under a floating rate would have a significant impact on the exchange rate. Under a target zone regime the instability of the exchange rate creates a much higher frequency of interventions and a much shorter lifetime than would be the case if the economy was affected only by monetary disturbances. This paper attempts to address this issue by analysing the lifetime of a target zone model with a richer underlying structure than is associated with the Krugman model. It is

${ }^{1}$ The ERM crisis provided a significant impetus to the development of second generation models. For examples see the paper by Ozkan and Sutherland (1994) and the volume edited by Cobham (1994). Obstfeld (1994) provides a overview of these second generation models. 
shown how for the identical target zone regime as was used in Dumas and Svensson (1994) the expected lifetime can in certain circumstances be very low.

The remainder of the paper is organized into the following sections. Section 2 outlines an extended version of the target zone model which is used in this paper. The extended target zone model provides a richer specification of the underlying fundamentals. This has the advantage of allowing for the explicit analysis of the effects of both real and monetary disturbances and for variations in the underlying structure of the model. This extended target zone model is due to Sutherland (1995). Section 3 uses the first passage time technique to calculate the expected lifetime of a target zone regime. Dumas and Svensson (1994) calculate the lifetime in a variety of scenarios. The purpose here is to focus on the effects of explicitly modelling real as well as monetary shocks and in varying the degree of interdependence of the real and monetary sectors. To do this it is necessary only to examine some particular case. It seems highly likely that there is some form of drift and as Dumas and Svensson show, this drift will be a major determinant of the lifetime. In addition some form of drift is central to most explanations of currency crises. Therefore, it seems natural to choose the situation in which a drift eventually causes the collapse of the target zone. As would be expected, it is briefly shown that the effects of changing the structure of the model readily extend to the simpler case of a drift-less fundamental. Section 4 contains the results.

\section{The model}

The Sutherland (1995) version of the target zone is defined by the following system of equations;

$$
\begin{aligned}
& y^{\mathrm{s}}=\alpha p+\varepsilon, \\
& y^{\mathrm{d}}=\eta\left(s-p+p^{*}\right)-\gamma i+\omega, \\
& m-p=\phi y-\lambda i-v, \\
& E \frac{(\mathrm{d} s)}{\mathrm{d} t}=i-i^{*}, \\
& \mathrm{~d} v=u_{v} \mathrm{~d} t+\sigma_{v} \mathrm{~d} z_{v}, \\
& \mathrm{~d} \varepsilon=u_{\varepsilon} \mathrm{d} t+\sigma_{\varepsilon} \mathrm{d} z_{\varepsilon}, \\
& \mathrm{d} \omega=u_{\omega} \mathrm{d} t+\sigma_{\omega} \mathrm{d} z_{\omega},
\end{aligned}
$$

where $y$ is the domestic output, $p$ is the price level, $m$ is the nominal money supply, $i$ is the nominal interest rate, $s$ is the nominal exchange rate, and $\varepsilon, \omega, v$ are the supply, demand and velocity disturbances, respectively. All variables except $i$ and $i^{*}$ are in logs and the asterisk denotes a foreign variable.

Eq. (1) is the aggregate supply curve where $\varepsilon$ represents a disturbance term. That aggregate supply responds positively to the price level in the long run implies that 
nominal wages are permanently fixed. This is an undesirable feature of the model but is not serious for the current paper. This is because the main results of the paper require aggregate supply to be unresponsive to movements in the price level ${ }^{2}$ so that in general it is assumed that $\alpha=0$. It is not assumed that the natural rate is unaffected by stochastic disturbances. Eq. (2) defines aggregate demand to be a function of the real exchange rate, nominal interest rate and the demand disturbance $\omega$. Eq. (3) is the standard money market equilibrium condition, where real money demand is determined by output, the nominal interest rate and the disturbance term $v$. Eq. (4) represents the uncovered interest parity condition. The disturbance terms $\varepsilon, \omega$ and $v$ are each assumed to follow independent Brownian motion processes with constant drifts.

The advantage of this model as compared to the Krugman model is the linkage between monetary and real sectors and the explicit inclusion of both real and monetary disturbances. The key parameters of the model are the slope of the aggregate supply curve, $\alpha$ and the real exchange rate elasticity of aggregate demand $\eta$. Structurally the model differs from the Krugman model in that it allows for a less than infinite real exchange rate elasticity of demand, $\eta<\infty$. This means the aggregate demand curve is downward sloping rather than horizontal. The importance of this parameter is that it is the main determinant of the degree of exchange rate variability in response to a real disturbance. If $\eta=\infty$ the nominal exchange rate is always defined by continuous purchasing power parity. If $\alpha=0$, output is fixed at the natural rate. These two conditions along with the assumption that there are no disturbances to aggregate supply can be used to obtain the Krugman form of the target zone model as a special case of the extended model. The extended model therefore allows a explicit analysis of the effects of various types of disturbance, both real and monetary, under scenarios in which the output is not fixed and purchasing power parity does not hold continuously.

The model is solved by defining a new fundamental variable which itself is a composite of the individual fundamentals. This is:

$$
g=\psi_{1}(m+v)+\psi_{2} \varepsilon+\psi_{3} \omega,
$$

where,

$$
\begin{aligned}
& \psi_{1}=\frac{-(\alpha+\eta)}{[\lambda(\alpha+\eta)+\gamma(1+\alpha \phi)]}, \\
& \psi_{2}=\frac{-(1-\phi \eta)}{[\lambda(\alpha+\eta)+\gamma(1+\alpha \phi)]}, \\
& \psi_{3}=\frac{+(1+\alpha \phi)}{[\lambda(\alpha+\eta)+\gamma(1+\alpha \phi)]},
\end{aligned}
$$

\footnotetext{
${ }^{2}$ A target zone version of the sticky price Dornbusch (1976) model has been developed by Miller and Weller (1991). It is desirable to use that model here but the dynamic adjustment of prices in the model makes it extremely difficult to analyse for multiple disturbances.
} 


$$
\psi_{4}=\frac{+(1+\alpha \phi) \eta}{[\lambda(\alpha+\eta)+\chi(1+\alpha \phi)]} .
$$

The fundamental $g$ follows a composite Brownian motion process where,

$$
\begin{aligned}
& \mathrm{d} g=\mu_{\mathrm{g}} \mathrm{d} t+\sigma_{\mathrm{g}} \mathrm{d} z_{\mathrm{g}}, \\
& \sigma_{\mathrm{g}}^{2}=\psi_{1}^{2} \sigma_{v}^{2}+\psi_{2}^{2} \sigma_{\varepsilon}^{2}+\psi_{3}^{2} \sigma_{\omega}^{2}, \\
& \mu_{\mathrm{g}}=\psi_{1} \mu_{v}+\psi_{2} \mu_{\varepsilon}+\psi_{3} \mu_{\omega} .
\end{aligned}
$$

The reduced form of the structural equations can then be written in terms of the composite fundamental as;

$$
s=-\frac{g}{\psi_{4}}+\frac{1 E(\mathrm{~d} s)}{\psi_{4} \mathrm{~d} t} .
$$

This equation has exactly the same reduced form as the Krugman target zone model and the solution for the exchange can now be obtained following the same procedure. Briefly, the first step is to assume that the exchange rate is free from arbitrary bubbles so that it can be written as a function of the fundamental only, $s=f(g)$. Ito's lemma is then applied to derive the expected rate of depreciation. These two expressions are then substituted back into Eq. (16) to obtain a second order ordinary differential equation in $f(g)$ which is solved in the standard way. ${ }^{3}$ This is:

$$
s=-\frac{g}{\psi_{4}}-\frac{\mu_{\mathrm{g}}}{\psi_{4}^{2}}+A_{1} \mathrm{e}^{\rho_{1} g}+A_{2} \mathrm{e}^{\rho_{2} g},
$$

where,

$$
\rho_{1}, \rho_{2}=\frac{-\mu_{\mathrm{g}} \pm\left(\mu_{\mathrm{g}}^{2}+2 \psi_{4} \sigma_{\mathrm{g}}^{2}\right)^{1 / 2}}{\sigma_{\mathrm{g}}^{2}} .
$$

The arbitrary constants are defined by requiring that the solution for the exchange rate is momentarily flat at the bands edges. This gives the inverse $\mathrm{S}$ shaped path shown in Fig. 1. This solution assumes parameter values which correspond to the Dumas and Svensson paper. These are, a bandwidth of $\pm 1.5 \%, \sigma_{\mathrm{g}}^{2}=1 \%$ per year, $\mu_{\varepsilon}=\mu_{\omega}=0, \mu_{v}=10 \%$ per year, $\lambda=1, \alpha=0$ and $\eta \rightarrow \infty$. For these parameters the band on the exchange rate results in the following limits for the band on the fundamental $a=-4.91 \%$ and $b=+8.99 \%$.

The exchange rate is free to move within the upper and lower limits, $\bar{s}$ and $\underline{s}$, respectively. The limits are defended using non-sterilized interventions to the money supply. The interventions are marginal and reflecting so that reserves fall at $\bar{s}$ and rise at $\underline{s}$. It also means the limits on the exchange rate and fundamental coincide. Because the interventions represent predictable movements in the money supply they

\footnotetext{
${ }^{3}$ For further details on the solution of this model see Sutherland (1995). Note that the model used here has been extended to incorporate drifts as well as Brownian motion shocks.
} 


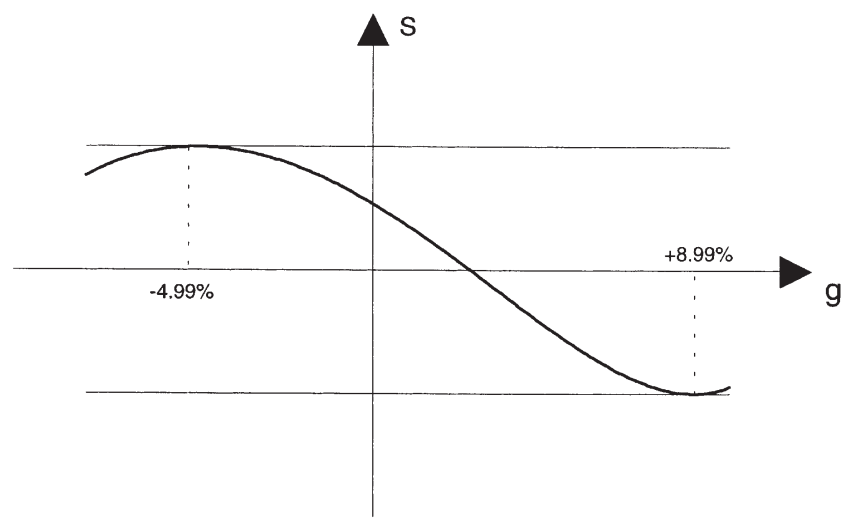

Fig. 1. A target zone with multiple disturbances.

cause the expected depreciation to point towards the middle of the band. That is, as the exchange rate approaches the upper limit, the expectation of a monetary contraction and exchange rate appreciation increases. This dampens the movement in the exchange rate relative to a movement in the fundamental. The reverse is true at the lower limit. The strength of this effect increases the closer the current rate is to either of the limits. This gives the exchange rate its inverse $\mathrm{S}$ shape. Once the exchange rate reaches either of the band limits, any further movement beyond the limit is offset by the combination of the marginal change in the money supply and the expected depreciation. This means that at the limits the exchange rate is approximately flat or rather that it 'smooth pastes' onto the edge of the band. This smooth pasting provides a condition which is used to obtain values for the arbitrary constants in (15). The major pictorial difference between this model and that of Krugman is that when graphed against the composite fundamental the $\mathrm{S}$ curve is negatively sloped, so that the limits on the exchange rate coincide with the opposite limits on the fundamental. In the Krugman model the $\mathrm{S}$ curve is positively sloped. The models are identical in that positive velocity shocks depreciate the exchange rate towards the upper edge of the band. However, positive velocity shocks reduce the composite fundamental which is the opposite of what happens in the Krugman model. Thus for velocity shocks to have the same effect the $\mathrm{S}$ curve is negatively and not positively sloped.

\section{The lifetime of the target zone}

In this section the method of Dumas and Svensson is used to derive the mean and density of the expected lifetime in the extended target zone model. This method is extremely neat. Assume that implicit constraints on monetary policy prevent the money supply, $M=D+R$ being reduced below some critical minimum $\bar{M}$. Further assume that domestic credit is held constant at this level. This means that the target zone will collapse if reserves fall to zero. The prospect of this triggers a buying 
attack which causes the target zone to collapse before reserves would have otherwise run to zero. Therefore, there is a critical lower level of the reserve stock at which the attack is triggered and the target zone collapses. In logs let this be $r=p$. The collapse occurs at the first instant log reserves fall to $p$. Since reserves only fall at $\bar{s}$ or equivalently when $g=a$ then the target zone collapses on the first occasion that $g=a$ and $r=p$. Dumas and Svensson also assume that the target zone is abandoned when reserves and the money supply become too large. Letting $r=q$ denote the critically high stock of $\log$ reserves means at the date of abandonment, $g=b$ and $r=q$.

The next step is to note that the fundamental $g$ follows a regulated Brownian motion process. The fundamental $g$ is regulated by changes in the money supply at the limits $a$ and $b$. If $-B_{1} r$ is subtracted from $g$ this results in an unregulated fundamental which depends only on the composite Brownian motion. That is,

$$
g-B_{1} r=B_{1} v+B_{2} \varepsilon+B_{3} \omega .
$$

It follows that as long as the target zone operates the unregulated fundamental is constrained to lie within the interval,

$$
a-B_{1} p<g-B_{1} r<b-B_{1} q .
$$

The target zone collapses on the first date that,

$$
g-B_{1} r=a-B_{1} p .
$$

The zone is abandoned on the first date that,

$$
g-B_{1} r=b-B_{1} q
$$

The lifetime of the target zone can now be equivalently modelled as the first passage time of the unregulated Brownian motion variable $g-B_{1} r$ to the limits given in (21a) or (21b). Formulas for a variety of scenarios are available in standard texts on stochastic processes, such as Cox and Miller (1965). To analyse the dependence of the survival time on the structure of the model it is only necessary to concentrate on some single case. For obvious reasons this is chosen to be a collapsing target zone, or rather hitting the critically low reserve level. It is straightforward to extend the results to the case of both critically high and low levels, although if it is assumed that there exists a yearly drift of $10 \%$ the results for critically high and low levels are virtually identical. The reason is that the probability of hitting the critical level in the opposite direction to the drift is virtually zero for the parameters chosen. For a smaller drift size this would not be true. It is straightforward to extend the analysis to the case of an abandonment caused by a critically high level of the money supply.

The probability density and expected survival time conditional upon the initial levels of $g$ and $r$ are:

$$
\begin{aligned}
& \phi(T \mid g, r)=\left(\frac{\left(a-\psi_{1} p\right)-\left(g-\psi_{1} r\right)}{\sigma_{\mathrm{g}}\left(2 \pi T^{3}\right)^{1 / 2}}\right) \exp \left(\frac{-\left(\left(a-\psi_{1} p\right)-\left(g-\psi_{1} r\right)-\mu_{\mathrm{g}} T\right)^{2}}{2 \sigma_{\mathrm{g}}^{2} T}\right), \\
& E[T \mid g, r]=\left(\left(a-\psi_{1} p\right)-\left(g-\psi_{1} r\right)\right) / \mu_{\mathrm{g}} .
\end{aligned}
$$




\section{Results}

The aim is to examine how varying the structure of the model and the source of disturbances affects the expected lifetime. As compared to the analysis of Dumas and Svensson the advantage is the richer specification of the underlying fundamental. In particular, the Krugman target zone model is a reduced from of the monetary model of exchange rates with output assumed to be fixed at a natural rate. The fundamental of the Krugman model is, strictly speaking, the stochastic money supply. However, the fundamental is usually interpreted in a more general way, and as in Dumas and Svensson a single stochastic variable is interpreted as representing the combined degree of real and monetary divergence. This assumes that all types of disturbance would lead to one for one movements in the exchange rate under a floating regime. This seems a fairly strong assumption even if output is fixed at a natural rate. It may be a very strong assumption for an economy which specializes in a narrow range of tradable products. A disturbance to the supply or demand for such a product might create a much greater degree of exchange rate instability than would a monetary disturbance of similar size. To examine this issue, Fig. 2 reproduces the density function simulated in Dumas and Svensson. We then examine how the shape of this function changes as the source of disturbances and the structure of the model are changed. In order to generate Fig. 2 the parameter values are chosen so that the extended form of the target zone model simplifies to the monetarist reduced form on which the Krugman model is based. The required values are: $\phi=1, \lambda=1, \gamma=0, \alpha=0, \eta \rightarrow \infty, p=0, q=200 \%, \mu_{\varepsilon}=\mu_{\omega}=0, \mu_{\nu}=10 \%$ per year, $\sigma_{\varepsilon}^{2}=\sigma_{\omega}^{2}=0$, $\sigma_{v}^{2}=1 \%$ per year, $g=(a+b) / 2$ and $r=(p+q) / 2$. The latter assumption gives an initial log excess reserve stock above the critical minimum of $r-p=100 \%$.

For these values the mean lifetime is approximately 10.7 years and the probability of collapse within 5 years is just $1.7 \%$. Trivially increasing the size of the drift or reducing the log excess of reserves reduces the mean lifetime. The mean lifetime can be decomposed into the time bought by excess reserves and the time bought by the bandwidth. With the initial values of the reserve stock and fundamental calculated at the mid-points the mean lifetime is:

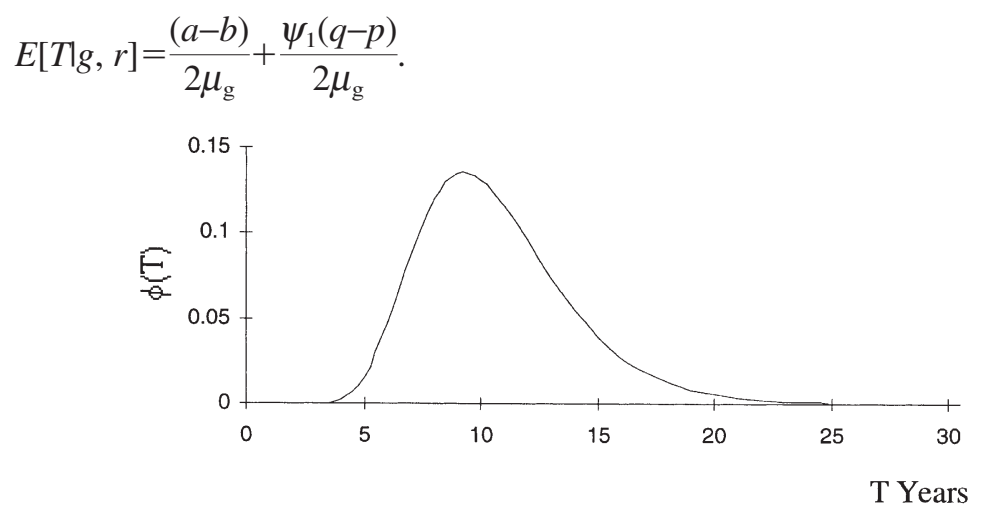

Fig. 2. $\phi\left[T \mid r, g, \mu_{v}=.1, \sigma_{v}=.1, \alpha=0, \eta \rightarrow \infty\right]$. 
The first term represents the lifetime bought by the bandwidth and the second term the time bought by excess reserves. For the parameter values consistent with the Krugman model this becomes:

$$
E[T \mid m+v, r]=\frac{(b-a)}{2 \mu_{v}}+\frac{(q-p)}{2 \mu_{v}} .
$$

Of the total mean lifetime of 10.7 years, 10 years are bought by excess reserves with the bandwidth of $\pm 1.5 \%$ adding only an additional $8 \frac{1}{2}$ months. Unless the band is extremely wide or if excess reserves are extremely low the choice of bandwidth is a relatively unimportant determinant of the expected lifetime. Even a fixed rate ( $b=a$ ) lasts for 10 years, although the drift of velocity is assumed to be $10 \%$ per year.

Fig. 2 serves as a baseline case. The remaining subsections examine how the zone's lifetime changes as the structure of the model is changed. In Section 4.1 the model is extended to see if allowing for real effects of monetary disturbances has any significant effects on the mean lifetime. Section 4.2 examines the relationship between the mean lifetime, real disturbances and the structure of the model. In varying the underlying structure of the extended target zone model the results focus on the elasticity of aggregate supply to the price level $\alpha$, and the elasticity of aggregate demand to the real exchange rate, $\eta$. The reason for focusing on these parameters can be seen from Eq. (24). The time bought by the excess stock of reserves is determined by $\left(\psi_{1} / 2 \mu_{\mathrm{g}}\right)$. This expression is free of $\lambda$ and $\gamma$. These parameters do affect the time bought by the bandwidth but this is only significant if the time bought by excess reserves is very low. The elasticity of the transactions demand for money does affect the time bought by excess reserves, particularly in the case of demand disturbances, but it seems unlikely that $\phi$ will deviate much from unity. Therefore, the focus of the analysis is on the two parameters that do affect the time bought by excess reserves and which conceivably vary greatly amongst countries. The driftless case and the effects of changing the values of $\lambda, \gamma$ and $\phi$ are briefly examined in Section 4.3.

\subsection{Monetary disturbances in the extended model}

With purely monetary disturbances the drift of the composite fundamental equals $\mu_{\mathrm{g}}=\psi_{1} \mu_{v}$ Substitution into (24) gives the mean lifetime in the extended model:

$$
E[T \mid g, r]=\frac{(b-a)}{2 \psi_{1} \mu_{v}}+\frac{(q-p)}{2 \mu_{v}} .
$$

The second term of (26) represents the time bought by excess reserves or alternatively the lifetime of a fixed rate. Under a fixed rate regime a monetary disturbance is offset by an equal and opposite change in reserves. Thus a downward drift in money demands of $10 \%$ per year creates a $10 \%$ per year drain on reserves. For an initial reserve stock $100 \%$ above the critical minimum the fixed rate lasts for 10 years. Because of the direct relationship between money disturbances and reserve changes this part of the lifetime is unaffected by any of the structural parameters of 


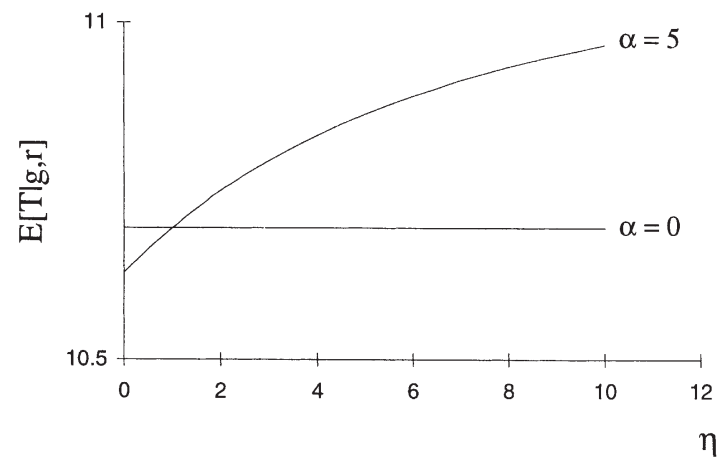

Fig. 3. $E\left[T \mid r, g, \mu_{v}=.1, \sigma_{v}=.1\right]$ and $\eta$.

the model. It therefore represents the minimum lifetime in the case of purely monetary disturbances. It follows that varying the structure of the model only alters the $8 \frac{1}{2}$ months of lifetime bought by the bandwidth.

Figs. 3 and 4 demonstrate that varying the parameters $\alpha$ and $\eta$ leads to only very small changes in the mean lifetime. For the changes shown the mean lifetime remains well within a range of 10-11 years. Specifically, Fig. 3 shows that unless output is fixed, $\alpha=0$ increasing the elasticity of aggregate demand to the real exchange rate, $\eta$, increases the mean lifetime. A positive monetary disturbance depreciates the nominal and, unless $\alpha=0$, the real exchange rate which raises goods and money demands. The larger is $\eta$ the bigger are the increases in goods and money demands to any given size of depreciation. Under the target zone regime the depreciation of $\pm 1.5 \%$ raises money demands by a larger amount the bigger is $\eta$ and this reduces the size of the remaining excess supply of money at $\bar{s}$. Thus raising $\eta$ reduces the number of marginal interventions which increases the mean lifetime. If output is fixed the real exchange rate is constant and equilibrium is restored by the combination of the rise in domestic prices and interventions at $\bar{s}$. This gives a mean lifetime of 10.7 years and corresponds to the strict form of monetary model on which the Krugman model is based.

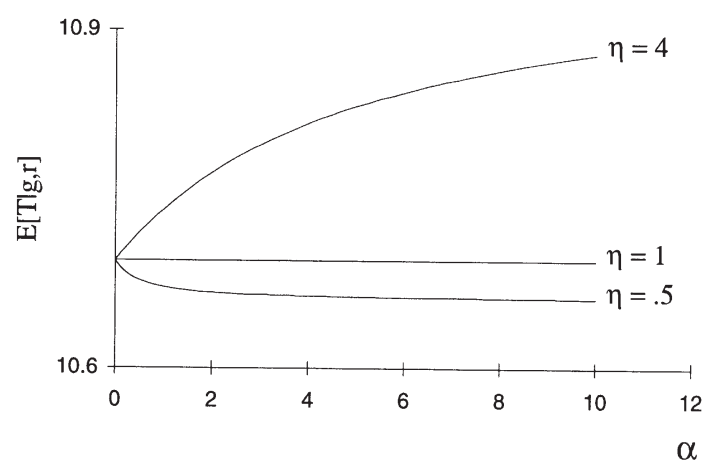

Fig. 4. $E\left[T \mid r, g, \mu_{v}=.1, \sigma_{v}=.1\right]$ and $\alpha$. 
Fig. 4 illustrates the effects of increasing the price elasticity of supply, $\alpha$. The effect on the lifetime is determined by the value of $\phi \eta$. This is easiest to understand by comparing the amount of exchange rate variability under a free float with a fixed rate of output against a situation in which output is not fixed. With a fixed rate of output, purchasing power parity holds and the nominal exchange rate depreciates by an amount which exactly matches to the original disturbance. If output is not fixed the price level rises by less than the nominal exchange rate so that the real exchange rate depreciates. In terms of money demand the reduction in the size of the increase in the price level raises money demand with an elasticity of one. However for a value of $\alpha$ other than zero the real exchange rate depreciation raises aggregate demand and output with an elasticity of $\eta$. The increase in output raises the demand for money with an elasticity of $\phi$. The overall elasticity of money demand to the real exchange rate is the product of the elasticity of aggregate demand to the real exchange rate and the elasticity of the transactions demand for money $\phi \eta$. Thus if $\phi \eta<1$ raising $\alpha$ reduces the increase in money demand in response to a real exchange rate depreciation, whilst if $\phi \eta>1$ the reverse is true. It follows that if $\phi \eta<1$ raising $\alpha$ increases the size of the depreciation caused by a monetary disturbance under a floating regime. In the target zone regime this means more frequent interventions and a reduced lifetime. The reverse is true if $\phi \eta>1$, whilst if $\phi \eta=1$ the mean lifetime is unaffected by the value of $\alpha$ and is equal to the baseline case of 10.7 years. Despite these effects on the time bought by the bandwidth the overall effect on the lifetime in the case of monetary disturbances is relatively minor.

\subsection{Real disturbances}

The previous subsection shows that when the disturbances only emanate in the money market the structure of the model is not an important determinant of the zone's lifetime. The model's structure is much more important when real disturbances are included. To illustrate this, consider the mean lifetime in the case of purely supply disturbances. Substituting for the values of $\mu_{\mathrm{g}}$ and $\psi_{1}, \psi_{2}$ into (24) and setting $\mu_{v}=\mu_{\omega}=0$ yields:

$$
E[T \mid g, r]=\frac{(a-b)}{2 \psi_{2} \mu_{\varepsilon}}+\frac{(\alpha+\eta)}{2(1-\phi \eta) \mu_{\varepsilon}}(q-p) .
$$

Varying the structural parameters, $\alpha, \eta$ and $\phi$ now alters the mean lifetime, through both the time bought by the bandwidth and the time bought by excess reserves. The reason that $\alpha$ and $\eta$ alter the time bought by excess reserves is that real disturbances indirectly affect the money market and exchange rate. In the case of monetary disturbances a one for one change in the money supply is sufficient to offset the disturbance. In the case of real disturbances the exchange rate and money supply must adjust to re-establish equilibrium in both the goods and money markets. This can require much larger movements in the exchange rate and money supply.

In calculating the mean lifetime in the case of supply disturbances it is assumed that $\mu_{\varepsilon}= \pm 10 \%$ per year. If $\phi \eta<1$ the exchange rate depreciates in response to a positive supply disturbance. In this case the drift is chosen to be $\mu_{\varepsilon}=+10 \%$ per year. 
However, if $\phi \eta>1$ a positive drift causes the exchange rate to appreciate. In order to generate a positive probability of collapse the drift is chosen to be $\mu_{\varepsilon}=-10 \%$ per year. The explanation for this is that a positive supply disturbance lowers the price level and raises output. These changes have opposing effects on money demand so that the exchange rate may either appreciate or depreciate. The extent to which the price level falls and output rises depends upon the value of $\eta$. If this elasticity is very large the aggregate demand curve is very flat and a very small real depreciation will raise aggregate demand significantly. It follows that with $\eta$ large most of the effect of a supply disturbance is concentrated in output with only a relatively small change in the price level. Conversely if $\eta$ is very low the fall in the price level is much larger and the increase in output much smaller. The increase in output raises money demand with an elasticity of $\phi$. This means a supply disturbance changes the transaction's demand for money with an elasticity of $\phi \eta$. On the other hand, the elasticity of money demand with respect to the price level is one. The overall effect on money demand depends upon a comparison of the two. In particular, if $\phi \eta<1$ the reduction in money demand from the fall in the price level dominates causing the exchange rate to depreciate. If $\phi \eta>1$, money demand rises and the exchange rate appreciates so that the drift in supply is chosen to be negative. Figs. 5 and 6 illustrate effects on the mean lifetime of changing the values of $\eta$ and $\alpha$.

As would be expected from the above discussion, Fig. 5 illustrates that increasing $\eta$ can either increase or decrease the mean lifetime, depending upon the value of $\phi \eta$. For very low values of $\eta$ the mean lifetime is very low. For example, if $\eta=0.1$ the mean lifetime is just 1.2 years. When $\phi \eta<1$, increasing $\eta$ increases the mean lifetime. For $\eta=0.25$ the mean lifetime is 3.6 years and for $\eta=0.5$ the mean lifetime is 10.8 years. As $\eta$ approaches unity the net effect of a supply disturbance on the money market become smaller and smaller, causing the mean lifetime to become infinitely long. When $\phi \eta=1$ the fall in the price level and the rise in output have perfectly offsetting effects on money demand. For larger values of $\eta$, such that $\phi \eta>1$ the drift of supply is chosen to be negative and increases in $\eta$ now reduce the mean lifetime. For example, when $\eta=1.5$ the mean lifetime is 32.4 years, when

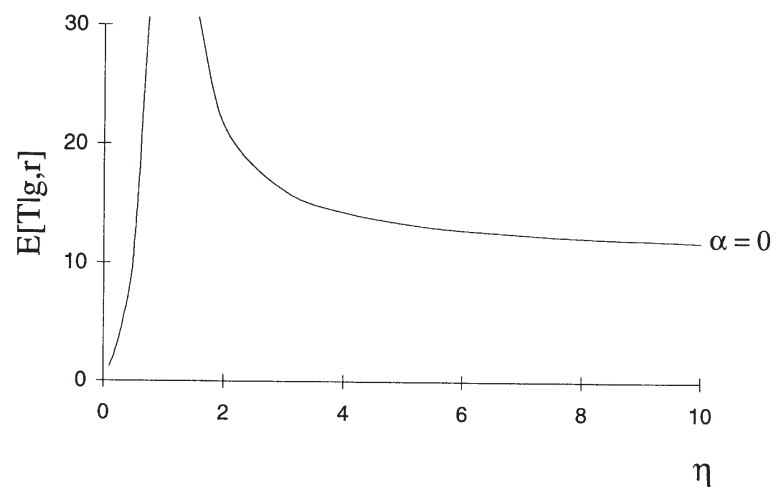

Fig. 5. $E\left[T \mid r, g, \mu_{\varepsilon}=.1, \sigma_{\varepsilon}=.1\right]$ and $\eta$. 


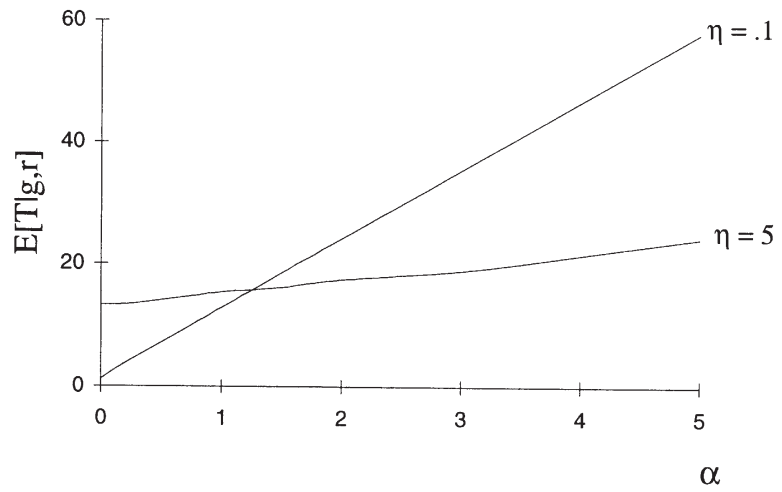

Fig. 6. $E\left[T \mid r, g, \mu_{\varepsilon}=.1, \sigma_{\varepsilon}=.1\right]$ and $\alpha$.

$\eta=10$ the mean lifetime is 11.8 years. There is however an asymmetry between the effects of raising $\eta$ when $\phi \eta<1$ and raising $\eta$ when $\phi \eta>1$. This occurs because whilst raising $\eta$ when $\phi \eta<1$ reduces the size of the original excess money supply it also increases the elasticity of aggregate demand to the real exchange rate. Both effects combine to raise the mean lifetime. If $\phi \eta \geq 1$ raising $\eta$ raises the size of the original excess money supply but it still raises the elasticity of aggregate demand to the real exchange rate. These have opposing effects on the mean lifetime which prevents the lifetime from falling to zero as $\eta \rightarrow \infty$.

Fig. 6 shows that unless $\eta \rightarrow \infty$, increasing $\alpha$ raises the mean lifetime. If output is not fixed, the fall in prices created by the original disturbance creates a contraction of domestic supply which reduces the effects of the original disturbance. For example, if $\eta=0.1$ and $\alpha=0$ the mean lifetime is 1.2 years. Increasing $\alpha$ to 0.25 raises the mean lifetime to 4.3 years and if $\alpha=0.5$ the mean lifetime is 7.2 years. As the value of $\eta$ is increased the mean lifetime becomes less sensitive to the value of $\alpha$. The reason is that $\alpha$ determines the contraction in supply that occurs as the price level falls. Raising the value of $\eta$ reduces the size of the fall in the price level which reduces the importance of $\alpha$. When $\eta \rightarrow \infty$ the aggregate demand curve is horizontal and all of the effect of a supply disturbance is concentrated in output.

The mean lifetime in the case of purely demand disturbances is;

$$
E[T \mid g, r]=\frac{(a-b)}{2 \psi_{3} \mu_{\omega}}-\frac{(\alpha+\eta)}{2(1+\alpha \phi) \mu_{\omega}}(q-p) .
$$

Figs. 7 and 8 map out the change in the mean lifetime as $\eta$ and $\alpha$ are increased.

A negative drift of $10 \%$ per year is chosen in order that the exchange rate depreciates. Fig. 7 illustrates the effects of increasing $\eta$. As with supply disturbances the mean lifetime is very sensitive to the value of $\eta$. If $\eta=0.1$ the mean lifetime is 1.29 years, if $\eta=0.25$ the mean is 2.9 years, 5.5 years if $\eta=0.5$ whilst if $\eta$ is 1 , so that $\phi \eta=1$, the mean lifetime is identical to the baseline case of 10.7 years. For larger values of $\eta$, such that $\phi \eta>1$, the mean lifetime exceeds the baseline case and as $\eta \rightarrow \infty$, demand disturbances have no effect at all. The importance of $\eta$ is easiest to 


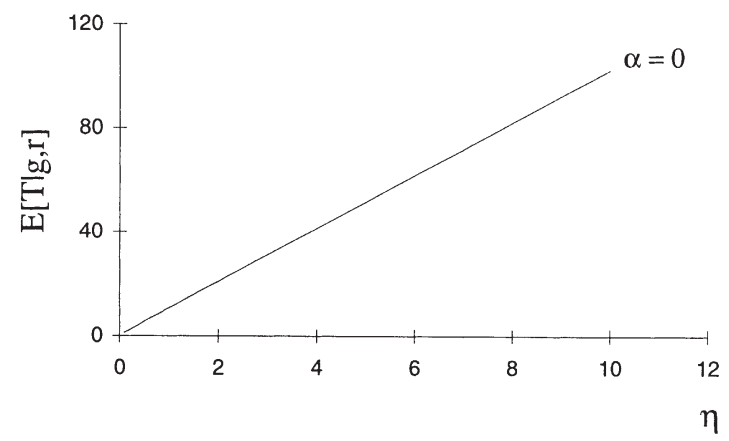

Fig. 7. $E\left[T \mid r, g, \mu_{\omega}=.1, \sigma_{\omega}=.1\right]$ and $\eta$.

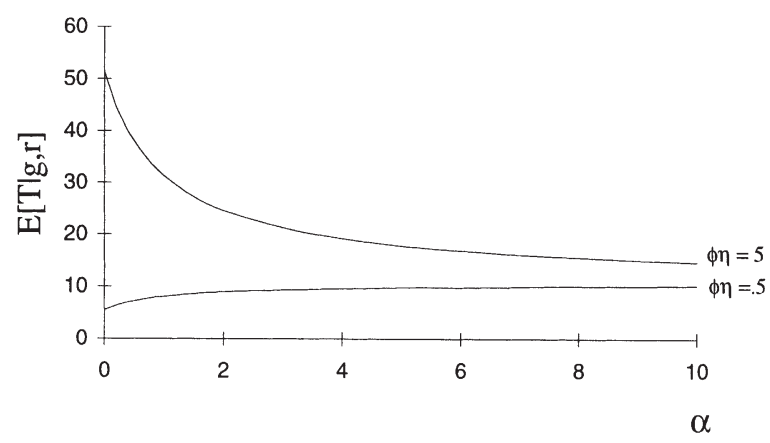

Fig. 8. $E\left[T \mid r, g, \mu_{\omega}=1, \sigma_{\omega \varepsilon}=.1\right]$ and $\alpha$.

understand by considering the effects of a demand disturbance under a pure free float. Under a pure free float a negative demand disturbance causes the nominal exchange rate to depreciate until demand returns to its previous level. Demand disturbances have no effect on prices and output under a floating regime. The extent of any depreciation is determined by the value of $\eta$. The larger is $\eta$ the more aggregate demand increases as the nominal and real exchange rate depreciates. Thus the larger is $\eta$ the smaller is the required depreciation. Under a target zone regime a low value of $\eta$ leads to frequent interventions and a low mean lifetime.

Fig. 8 maps out the effects of increasing $\alpha$. When $\alpha \eta<1$ increasing $\alpha$ raises the mean lifetime, whilst if $\phi \eta>1$ raising $\alpha$ reduces the mean lifetime. With $\alpha>0$ a negative demand disturbance will reduce both prices and output. The extent of these falls depends on $\eta$. As the price level falls the real exchange rate depreciates which offsets the original disturbance. That is, the value of $\eta$ will determine how much output falls relative to the price level. Given the value of $\alpha$ the larger is $\eta$ the smaller is the fall in the price level relative to output. The fall in the price level reduces money demand with an elasticity of one. The fall in output reduces money demand with an elasticity of $\phi$. With the fall in price level reducing output with elasticity of $\eta$, it follows that the a fall in output reduces money demands by $\phi \eta$ relative to a fall in the price level. Thus if $\phi \eta=1$ the money demand effects of a demand disturb- 


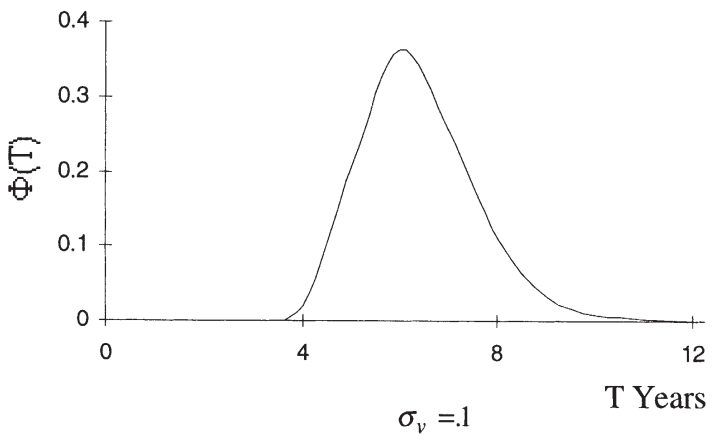

Fig. 9. $\phi\left[T \mid r, g, \alpha=0, \eta=.33, \sigma_{v}=.1\right]$.

ance have an elasticity of unity irrespective of whether output or the price level varies. In this case the excess money supply exactly matches the disturbance and this defines the size of the contraction in reserves that must occur at $\bar{s}$. It also defines the response to the depreciation of $s$ to $\bar{s}$. It follows that if $\phi \eta<1$ raising $\alpha$ raises the mean lifetime, whilst if $\phi \eta>1$ it reduces the mean lifetime. The fact that the lifetime is increased when $\alpha$ rises modifies the above results. For instance, when $\eta=0.25$ and $\alpha=0$ the mean lifetime is 2.9 years. Raising $\alpha$ to 0.25 raises the mean lifetime to 4.4 years and if $\alpha=0.5$ the mean lifetime is 7.24 years.

The results illustrate that for low values of $\alpha$ and $\eta$ the mean lifetime is significantly reduced if the disturbances emanate from real sources. On the other hand, the mean lifetime is significantly increased if $\alpha$ and $\eta$ are large and the primary source of disturbance is aggregate demand. Figs. 9 and 10 simulate the density function for the more interesting case when $\alpha$ and $\eta$ are low and when all types of disturbance occurs. Specifically, it is assumed that $\alpha=0, \eta=1 / 3, \mu_{v}=\mu_{\varepsilon}=0.03333$ and $\mu_{\omega}=-0.03333$. In terms of exchange rate depreciation this gives a combined real and monetary drift of $10 \%$ per year. ${ }^{4}$

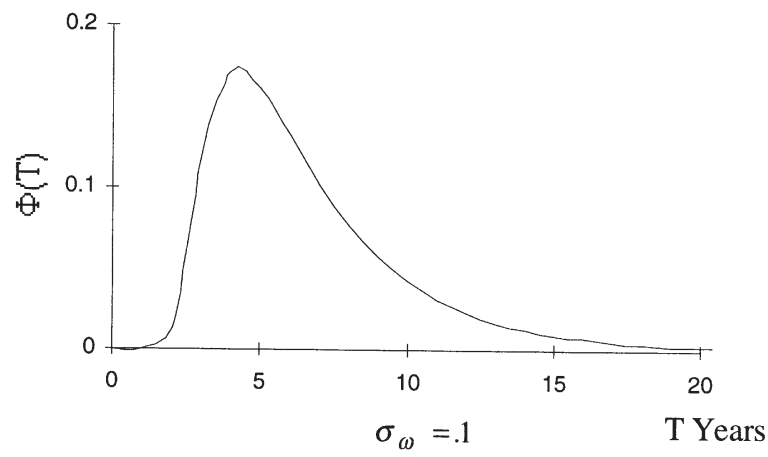

Fig. 10. $\phi\left[T \mid r, g, \alpha=0, \eta=.33, \sigma_{\omega}=.1\right]$.

\footnotetext{
${ }^{4}$ The composite drift is $\mu_{\mathrm{g}}=-23 \%$.
} 
In generating Fig. 9 it is assumed that $\sigma_{v}=0.1$, whilst for Fig. 10 it is assumed that $\sigma_{\omega}=0.1$. All other parameter values are as previously assumed which gives a variance of the composite fundamental of $\sigma_{\mathrm{g}}^{2}=0.01$ if $\sigma_{v}=0.1$, and $\sigma_{\mathrm{g}}^{2}=0.09$ if $\sigma_{\omega}=0.1$. This alters the shape of the density function in the usual way. The mean lifetime is fairly short, around $5 \frac{1}{2}$ years in both cases. The probability of collapse within 5 years is quite high at just under $60 \%$ in both cases. However, the larger variance associated with demand disturbances leads to a much larger probability of short and long lifetimes in the case of $\sigma_{\omega}=0.1$. In particular, if $\sigma_{v}=0.1$ there is a probability of collapse within 3 years of just $2 \%$. For $\sigma_{\omega}=0.1$ there is a $30 \%$ chance of collapse within 3 years and a $14 \%$ chance of collapse within 2 years. The probability of a lifetime over 10 years is similarly increased.

\subsection{The drift-less case and other factors}

Thus far the results concentrate solely on the collapse of a one-sided zone when the fundamental exhibits a drift which pushes the exchange rate towards the upper limit of the target zone. This means that the central bank loses reserves with a higher probability than it gain reserves. If the fundamental is drift-less these probabilities are equal and the lifetime will clearly be higher. The previous results regarding the structure of the model still apply though. For example, the mean lifetime of a twosided process $^{5}$ is given by:

$$
E(T \mid g, r)=\frac{\left[\left(g-\psi_{1} r\right)-\left(a-\psi_{1} p\right)\right]\left[\left(b-\psi_{1} q\right)-\left(g-\psi_{1} r\right)\right]}{\sigma_{\mathrm{g}}^{2}} .
$$

Choosing the parameter values to reproduce the Krugman model, as per Section 4, gives the Dumas and Svensson result that the mean lifetime is almost 120 years. However, as with the drift case the mean lifetime is considerably lower when the disturbance is in the real side of the economy and when the elasticity of demand to the real exchange rate is low. For example, if $\sigma_{\omega}=0.1$ and $\eta=1 / 2$ the mean lifetime is approximately 30 years, whilst if $\eta=0.25$ it is just 8.4 years even in the unlikely case that the fundamental exhibits no form of drift. As with the previous results the reason is that changing the structure of the model alters the moments of the composite fundamental. For instance, under the Krugman structure a monetary shock of $10 \%$ per year corresponds to a variance of $1 \%$ per year. With $\eta=1 / 3$ and $\sigma_{\omega}=0.1$ the variance of the composite fundamental is $9 \%$ per year.

In the previous results the parameters $\lambda, \gamma$ and $\phi$ were held constant. The parameters $\lambda$ and $\gamma$ measure the elasticities of money demand and aggregate demand to the domestic nominal interest rate. These parameters do not affect the time bought by excess reserves but they are key determinants of the stabilizing property of the

5 See Karatzas and Shreve (1988, p. 99). In the case of a drift-less fundamental and a one-sided process it is possible that the target zone would never collapse. Therefore, to generate a positive probability of collapse it is necessary to consider a two-sided process, that is both collapse and abandonment. 
zone. The key feature of the target zone model is that movements in the exchange rate create offsetting movements in the expected rate of depreciation and nominal interest rate. A depreciation creates a falling interest rate which raises money demand directly with an elasticity of $\lambda$, and indirectly through aggregate demand with an elasticity which increases in $\gamma$. It follows that raising the values of $\lambda$ and $\gamma$ enhances the amount of stability generated by the zone which increases the zone's lifetime.

Fig. 11 illustrates the effects of increasing $\lambda$ on the time bought by the bandwidth. The parameter values are assumed to be identical to those that were used to generate the densities in Figs. 9 and 10. Two possible bandwidths are assumed, a narrow zone of $\pm 1.5 \%$ and a wide zone of $\pm 15 \%$. For the narrow zone the time bought by the bandwidth is, for any realistic value of $\lambda$, fairly small. The wider zone buys approximately 1.3 years when $\lambda=1$, rising to 3 years when $\lambda=10$. In addition, if $\gamma$ is increased the time bought by the bandwidth will be higher. For example, if $\lambda=3$ and $\gamma=0$ the time bought by the bandwidth is 1.9 years; if $\gamma=1 / 2$ this rises to 2.21 years, 2.48 years when $\gamma=1$ and 3.35 years when $\gamma=5$. In all cases, for the values assumed the time bought by excess reserves is almost exactly equal to 5 years. Values of $\lambda=3$ and $\gamma=1 / 2$ do not seem unrealistic, in which case the time bought by the bandwidth amounts to over $30 \%$ of the mean lifetime.

The parameter $\phi$ represents the elasticity of the transaction's demand for money. This parameter has been held constant at unity throughout for two reasons. Firstly, it seems unlikely that $\phi$ will be anything other than unity except perhaps in the very short run. Secondly, it only affects the lifetime in the case of money and demand disturbances if the aggregate supply curve is non-vertical. Again this would give the model some undesirable long run properties. In the case of supply disturbances raising $\phi$ will increase in the size of the initial excess money supply or demand which is created. Thus raising $\phi$ will lower the mean lifetime. Nonetheless it is hard to imagine that $\phi$ could take on a value which is significantly different from unity and certainly not a value which is significantly greater.

The results of Sections 4.1 and 4.2 suggest that currency crises are at least as likely to be triggered by real disturbances as by a monetary disturbance. However, it is evident that the size of the real and monetary drifts might be quite different. In particular, the traditional Krugman (1979) model of a currency crisis focuses on the

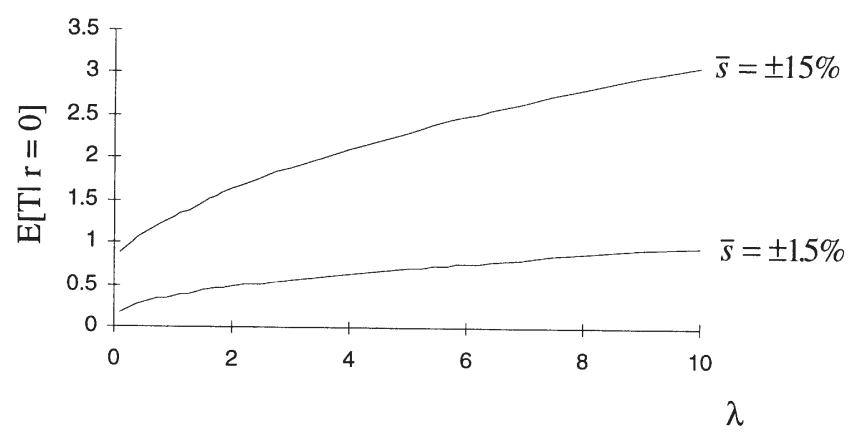

Fig. 11. Time bought the bandwidth and $\lambda$. 


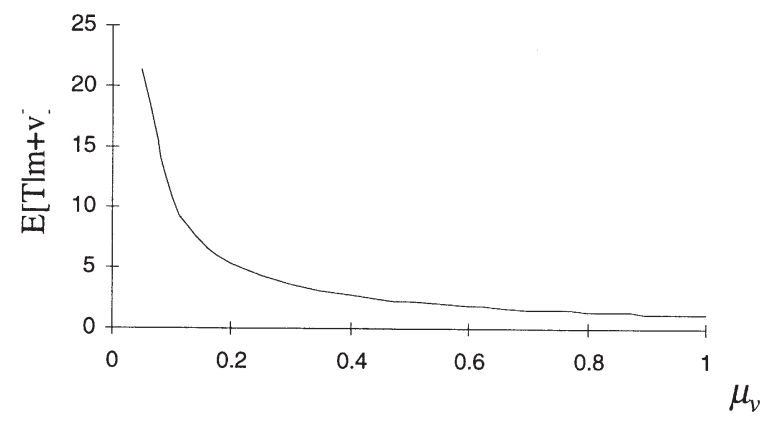

Fig. 12. $E[T \mid r, g, \alpha=0, \eta \rightarrow \infty]$ and $\mu_{v}$.

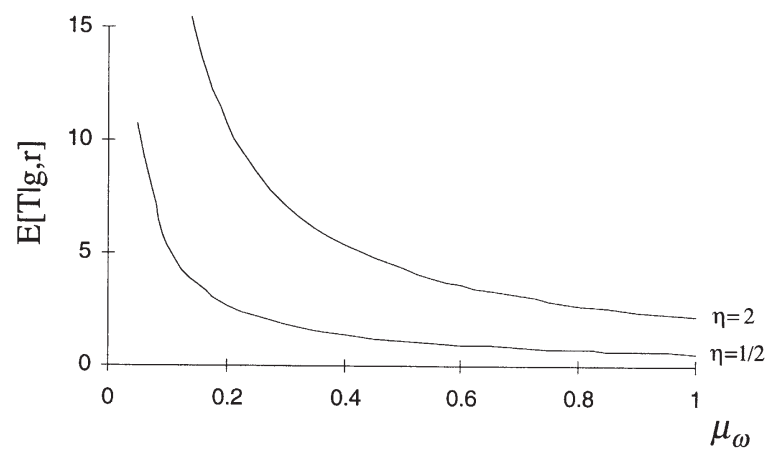

Fig. 13. $E[T \mid r, g, \alpha=0, \eta \rightarrow \infty]$ and $\mu_{\omega}$.

role of budget deficits and credit creation. The model is intended to represent a situation in which a large and presumably growing budget deficit leads to a high rate of credit creation and a rapid loss of reserves. In such a situation a much larger monetary drift than $10 \%$ per year is perfectly feasible. Fig. 12 provides a plot of the mean lifetime against the size of a monetary drift in the baseline case. Fig. 13 provides a plot of the mean lifetime against the drift in aggregate demand, assuming that aggregate supply is fixed at the natural rate. In the case of demand disturbances it is also assumed that either $\eta=0.5$ or $\eta=2$. Roughly speaking, increasing the size of the drift halves the mean lifetime in the case of monetary disturbances. The same is roughly true for demand disturbances. In addition, halving the value of $\eta$ approximately halves the mean lifetime for any given size of drift in demand.

\section{Conclusions}

This paper extends earlier work by Dumas and Svensson which examines the lifetime of an exchange rate target zone as a function of limited reserves. Underlying the modelling is the assumption that implicit constraints prevent the exclusive focus of monetary policy on the defence of the target zone. In these circumstances the 
lifetime of the target zone will depend upon the size of the reserve stock. Dumas and Svensson find the main determinants of the lifetime to be the size of the drift of fundamentals and the size of the reserve stock available to defend the target zone. In Dumas and Svensson a single drift term is interpreted as a the combined degree of real and monetary divergence. This paper uses an extended version of the target zone model to examine how variations in the underlying fundamentals which define this drift affect the lifetime of the target zone. The central result is that if the main source of disturbances is the goods market the lifetime of the target zone can, under certain circumstances, be much lower than was found by Dumas and Svensson. The reason for this is that unlike monetary disturbances real disturbances cannot always be offset by a one for one change in the domestic money supply. When real disturbances occur their effect on the money market and lifetime varies greatly as the structure of the underlying fundamental is changed. In particular, if aggregate demand is very unresponsive to the real exchange rate and if output is fixed at the natural rate the expected lifetime is very low. Under these circumstances restoring equilibrium in the goods market following a negative disturbance to aggregate demand requires a very large depreciation of the real exchange rate. With the nominal exchange rate bounded by the upper limit of the target zone the only way to restore equilibrium is if the domestic price level falls by a equivalently large amount. Therefore, a demand disturbance creates a much larger monetary disturbance and a correspondingly rapid depletion of the reserve stock. The lifetime of the zone is low only if the elasticity of aggregate demand to the real exchange is very low. Whilst really low values seem implausible in the medium to long run, such low values do not seem implausible over a short time period in which case the lifetime of the zone would indeed be very low.

The model assumes that there are implicit constraints which prevent the exclusive focus of monetary policy on the defence of the target zone. This corresponds to the first generation speculative attack models originated by Krugman (1979). A later class of models following Obstfeld (1986) have begun to make explicit these constraints. An interesting topic of future research would be to analyse the expected lifetime of a target zone in these second generation speculative attack models. A possible avenue of doing so might involve upper and lower and bounds on the movement of unemployment from some initial level.

\section{Acknowledgements}

The author is grateful for helpful comments from Alan Sutherland and to an anonymous referee. The usual disclaimer applies.

\section{References}

Cobham, D. (Ed.), 1994. European Monetary Upheavals. Manchester University Press, Manchester, UK. Cox, D.R., Miller, H.D., 1965. The Theory of Stochastic Processes. Chapman and Hall, London. 
Dornbusch, R., 1976. Expectations and exchange rate dynamics. Journal of Political Economy 84, 1161-1176.

Dumas, B., Svensson, L.E.O., 1994. How long do unilateral target zones last? Journal of International Economics 36, 467-481.

Flood, R.P., Garber, P.M., 1984. Collapsing exchange rate regimes: some linear examples. Journal of International Economics 17, 1-13.

Karatzas, I., Shreve, S., 1988. Brownian Motion and Stochastic Calculus. Springer-Verlag, New York.

Krugman, P., 1979. A model of balance of payments crises. Journal of Money, Credit and Banking 11, 311-325.

Krugman, P., 1991. Target zones and exchange rate dynamics. Quarterly Journal of Economics 106, $669-682$.

Miller, M., Weller, P., 1991. Exchange rate bands with price inertia. Economic Journal 101, 1380-1399.

Obstfeld, M., 1986. Rational and self-fulfilling balance of payments crises. American Economic Review 76, 102-138.

Ozkan, F.G., Sutherland, A., 1994. A model of the ERM Crises. CEPR Working Paper No. 879.

Sutherland, A., 1995. Monetary and real shocks and the optimal target zone. European Economic Review 39, 161-172. 